\title{
Effect of coexisting vascular disease on long-term risk of recurrent events after TIA or stroke
}

Marion Boulanger, MD, Linxin Li, MD, DPhil, Shane Lyons, MD, Nicola G. Lovett, MBBS, Magdalena M. Kubiak, MBBS, MRCP, MD, Louise Silver, DPhil, Emmanuel Touzé, MD, PhD, and Peter M. Rothwell, FMedSci, on behalf of the Oxford Vascular Study

Neurology ${ }^{\circledR}$ 2019;93:e695-e707. doi:10.1212/WNL.0000000000007935

\author{
Correspondence \\ Prof. Rothwell \\ peter.rothwell@ \\ nden.ox.ac.uk
}

\begin{abstract}
\section{Objective}

To determine whether patients with TIA or ischemic stroke with coexisting cardiovascular disease (i.e., history of coronary or peripheral artery disease) are still at high risk of recurrent ischemic events despite current secondary prevention guidelines.
\end{abstract}

\section{Methods}

In a population-based study in Oxfordshire, UK (Oxford Vascular Study), we studied consecutive patients with TIA or ischemic stroke for 2002-2014. Patients were treated according to current secondary prevention guidelines and we determined risks of coronary events, recurrent ischemic stroke, and major bleeding stratified by the presence of coexisting cardiovascular disease.

\section{Results}

Among 2,555 patients (9,148 patient-years of follow-up), those ( $\mathrm{n}=640 ; 25.0 \%)$ with coexisting cardiovascular disease (449 coronary only; 103 peripheral only; 88 both) were at higher 10 -year risk of coronary events than those without (22.8\%, 95\% confidence interval 17.4-27.9; vs 7.1\%, 5.3-8.8; $p<0.001$; age- and sex-adjusted hazard ratio [HR] 3.07, 2.24-4.21) and of recurrent ischemic stroke $(31.5 \%, 25.1-37.4$; vs $23.4 \%, 20.5-26.2$; $p=0.0049$; age- and sexadjusted HR 1.23, 0.99-1.53), despite similar rates of use of antithrombotic and lipid-lowering medication. However, in patients with noncardioembolic TIA/stroke, risk of extracranial bleeds was also higher in those with coexisting cardiovascular disease, particularly in patients aged $<75$ years (8.1\%, 2.8-13.0; vs 3.4\%, 1.6-5.3; $p=0.0050$; age- and sex-adjusted HR 2.71, 1.16-6.30), although risk of intracerebral hemorrhage was not increased (age- and sex-adjusted HR 0.36, 0.04-2.99).

\section{Conclusions}

As in older studies, patients with TIA/stroke with coexisting cardiovascular disease remain at high risk of recurrent ischemic events despite current management. More intensive lipidlowering might therefore be justified, but benefit from increased antithrombotic treatment might be offset by the higher risk of extracranial bleeding. 


\section{Glossary}

DWI = diffusion-weighted imaging; HR = hazard ratio; LDL = low-density lipoprotein; $\mathbf{M I}=$ myocardial infarction; $\mathbf{N}-$ STEMI = non-ST-elevation; OXVASC = Oxford Vascular Study; PCSK-9 = proprotein convertase subtilisin/kexin type 9; STEMI $=$ ST-elevation; TOAST $=$ Trial of Org 10172 in Acute Stroke Treatment.

Risk of recurrent vascular events in patients with TIA and stroke remains substantial, and there is a need for improved secondary prevention of stroke and also prevention of coronary events. ${ }^{1,2}$ Antiplatelet monotherapy is currently standard for long-term secondary prevention, and there is evidence in certain subsets of patients that anticoagulation is associated with a high risk of intracerebral hemorrhage. ${ }^{3,4}$ Patients with TIA/stroke with coexisting stable atherosclerotic cardiovascular disease (coronary or peripheral artery disease) have been shown previously to be at high absolute risk of recurrent stroke and coronary events. ${ }^{5-7}$ However, the absolute risk of coronary events after TIA/stroke has decreased over the last few decades, ${ }^{2}$ and there are relatively few recent data on the overall residual vascular risk in patients with TIA/stroke on current guideline-based management, particularly on longterm follow-up. Moreover, data on the long-term risk of major bleeding with antithrombotic therapies based on the presence of coexisting cardiovascular disease are scarce. If TIA/stroke patients with coexisting stable atherosclerotic cardiovascular disease still have a high absolute risk of recurrent ischemic stroke or coronary events, then more intensive secondary prevention might result in a sufficient reduction in ischemic events to counterbalance any increased risk of bleeding or increased cost of more intensive treatment.

In patients with stable atherosclerotic cardiovascular disease, the COMPASS trial reported a 50\% relative reduction in risk of ischemic stroke on rivaroxaban plus aspirin vs aspirin alone, albeit with a $70 \%$ increased risk of major bleeding. ${ }^{8} \mathrm{COM}$ PASS excluded patients with recent TIA or stroke, but the subgroup of patients with carotid artery disease did benefit from combination treatment. ${ }^{9}$ Moreover, the annual risk of stroke was $<1 \%$ in the aspirin-alone group, which is lower than reported after TIA and ischemic stroke in routine practice, ${ }^{10}$ particularly in patients with coexisting coronary or peripheral artery disease. ${ }^{6}$ In patients with recent cryptogenic stroke, the NAVIGATE ESUS trial reported no reduction in risk of recurrent ischemic stroke on rivaroxaban vs aspirin, but a higher risk of intracerebral hemorrhage. ${ }^{3}$ However, cryptogenic patients with TIA/stroke have a low prevalence of coexisting cardiovascular disease, have a relatively low absolute risk of recurrent ischemic stroke, ${ }^{11}$ and may be at higher risk of intracerebral hemorrhage. ${ }^{12-14}$

Patients with TIA and ischemic stroke also have a substantial long-term risk of myocardial infarction (MI) and cardiac death, ${ }^{15}$ and there is also increasing evidence that tighter control of cholesterol levels than the current guideline target (low-density lipoprotein $[\mathrm{LDL}]<2.6 \mathrm{mmol} / \mathrm{L}$ ) may be necessary, ${ }^{16}$ with randomized controlled trials of LDL reduction to $<1.8 \mathrm{mmol} / \mathrm{L}$ ongoing. ${ }^{17}$ Trials of new cholesterollowering therapies, proprotein convertase subtilisin/kexin type 9 (PCSK-9) inhibitors, ${ }^{18-20}$ also focused on populations with stable cardiovascular disease and reported significant reductions in LDL cholesterol and in MI, as compared with placebo, in patients on high-dose statins with LDL level $>1.8$ $\mathrm{mmol} / \mathrm{L}$. There was less evidence for a reduction in risk of ischemic stroke with PCSK-9 inhibitors, ${ }^{19,20}$ but dyslipidemia is estimated to account for a higher proportion of the risk of coronary events than of the risk of ischemic stroke. ${ }^{21,22}$ However, although PCSK-9 inhibitors reduced the relative risk of MI by $27 \%$ in the Further Cardiovascular Outcomes Research With PCSK9 Inhibition in Subjects With Elevated Risk (FOURIER) trial ${ }^{19}$ and by $13 \%$ in the Randomized Evaluation of the Effects of Anacetrapib Through Lipidmodification (REVEAL) trial, ${ }^{20}$ the drugs are very costly ${ }^{23}$ and may only be cost-effective in high-risk subgroups. The annual risk of MI was only $1 \%-2 \%$ in the trials, which is again lower than reported after TIA and ischemic stroke in patients with coexisting coronary or peripheral artery disease. ${ }^{6}$

Given the potential that more intensive antithrombotic treatment or lipid-lowering might reduce the long-term risk of recurrent stroke and coronary events in patients with TIA or stroke with coexisting stable cardiovascular disease, we aimed to determine the prognosis of this subgroup on current standard preventive treatment for both recurrent ischemic events and for major bleeding. Up-to-date estimates of event rates will help clinicians assess potential benefits of more intensive treatment and facilitate the planning of future trials.

\section{Methods}

\section{Study population}

The Oxford Vascular Study (OXVASC) is an ongoing population-based study of the incidence and outcome of all acute vascular events. ${ }^{24}$ The study population comprised all 92,728 individuals, irrespective of age, registered with 100 family physicians in 9 general practices in Oxfordshire, UK. In Oxfordshire, it is estimated that $97.1 \%$ of the true residential population is registered with a primary care practice, the majority of nonregistered individuals being young adults. The analysis reported here included consecutive cases, irrespective of age, with a first TIA or ischemic stroke from April 1, 2002, to March 31, 2014, with follow-up until September 30, 2014. Information on major extracranial bleeds was only available in cases recruited from April 1, 2002, to March 31, 2012, with follow-up until March 31, 2013. 
The OXVASC study design has been described in detail previously. ${ }^{24}$ Briefly, multiple overlapping methods of hot and cold pursuit were used to achieve near complete ascertainment of all individuals with TIA or stroke. ${ }^{25,26}$ These include the following: (1) a daily, rapid access TIA and stroke clinic to which participating general practitioners and the local emergency department refer individuals with suspected TIA or minor stroke; (2) daily searches of admissions to the medical, stroke, neurology, and other relevant wards; (3) daily searches of the local emergency department attendance register; (4) daily searches of in-hospital death records via the Bereavement Office; (5) monthly searches of all death certificates and coroner's reports for out-of-hospital deaths; (6) monthly searches of general practitioner diagnostic coding and hospital discharge codes; (7) monthly searches of all brain and vascular imaging referrals. Patients gave written informed consent after an event or assent was obtained from a relative for patients who were unable to provide consent. OXVASC was approved by our local research ethics committee.

Standard WHO definitions were used for TIA and stroke, which have been reported previously. ${ }^{24,25}$ All cases were investigated according to current guidelines and reviewed by a senior neurologist with stroke expertise (P.M.R.) in order to ensure consistency over time. Differentiation between TIA and stroke was based on the WHO 24-hour cutoff for symptom duration (i.e., events were classified as TIA even if diffusion-weighted imaging [DWI] was positive if symptoms resolved within 24 hours) and we have kept the same definition over time. TIA/stroke etiology was categorized by P.M.R. according to the modified Trial of Org 10172 in Acute Stroke Treatment (TOAST) criteria (table e-1, doi. org/10.5061/dryad.t4r1n64). ${ }^{27}$ Patients were prescribed standard secondary prevention medication according to contemporary guidelines, including antiplatelet or anticoagulant treatment, as appropriate, blood pressure-lowering medication, and statin treatment to achieve a target of blood pressure $<140 / 90 \mathrm{~mm} \mathrm{Hg}$ and $\mathrm{LDL}<2.6 \mathrm{mmol} / \mathrm{L}$, both at baseline and at the time of study follow-up visits. It was our policy in patients with coexisting cardiovascular disease who were on antiplatelet treatment before the TIA/stroke to continue dual therapy if already on dual (e.g., after recent stent) for as long as previously indicated and to add clopidogrel for 1 month only if on monotherapy beforehand, but revert to monotherapy after 1 month. Anticoagulation was also continued or started when clinically indicated, but was not used preferentially in patients with coexisting cardiovascular disease.

Demographic data, atherosclerotic risk factors (male sex, history of hypertension, diabetes mellitus, hypercholesterolemia, smoking [i.e., ex or current smoker], family history of premature MI [defined as MI occurring in a first-degree relative, siblings, or parents, aged $\leq 55$ years if male or aged $\leq 65$ years if female]), previous atrial fibrillation, prior coronary artery disease, prior peripheral artery disease, and medication were collected from face-to-face interviews and cross-referenced with primary care records. Renal insufficiency was defined as glomerular filtration rate $<30 \mathrm{~mL} /$ min, calculated by the MDRD equation, ${ }^{28}$ using the creatinine level closest to the date of TIA/stroke. Patients were classified as having a prior coronary artery disease if they had at least one of the following: previous MI, unstable angina, angina, previous percutaneous coronary intervention, or coronary artery bypass graft surgery. Prior peripheral artery disease was defined as having at least one of the following: previous acute limb ischemia, critical limb ischemia, intermittent claudication, previous angioplasty or stenting, peripheral arterial bypass graft, or amputation. For intermittent claudication, the diagnosis was based on the judgement of the study clinician based on both prior medical records and symptoms.

All patients were followed up face-to-face at 1, 6, 12, 60, and 120 months by a study nurse or physician and subsequent vascular events identified and risk factor control evaluated and treatment revised as necessary. For patients who had moved out of the study area, telephone follow-up was done, and patients with dementia were followed up via a carer or by assessment in a nursing home. All recurrent events that presented to medical attention would also be identified by ongoing daily case ascertainment of all acute vascular events in the study population. All study patients were also notified to the UK Office of National Statistics such that all deaths were reported back to the study with causes. Deaths were also identified by regular review of primary care records and by regular contact with the Coroner's Office to ascertain out-ofhospital deaths. If a recurrent nonfatal vascular event was suspected, the patient was reassessed face-to-face by a study physician.

MI was defined using standard criteria. ${ }^{29}$ Sudden cardiac death was defined using recommendations in Epidemiology and Clinical Research Studies and required a definite history of preceding symptoms consistent with acute coronary ischemia, or postmortem evidence of either substantial coronary atherosclerosis or acute thrombosis, or a documented MI during the previous 28 days. ${ }^{30}$ Fatal MI was defined as any death within 30 days of a documented MI. Definitions of MI and sudden cardiac death remained unchanged over the period. We collected information on MI subtype (ST-elevation [STEMI] and non-ST-elevation MI [N-STEMI]). Recurrent ischemic stroke was defined as new neurologic deficit fitting the definition for ischemic stroke occurring after a period of neurologic stability or improvement. Major bleeds were defined according to the Clopidogrel in Unstable Angina to Prevent Recurrent Events (CURE) criteria and were bleeds that were fatal or substantially disabling with persistent sequelae, symptomatic intracranial, intraocular bleeds leading to significant loss of vision, or bleeds requiring transfusion of $\geq 2$ units of blood, use of IV inotropic agents, or necessitating a surgical intervention (appendix e-1, doi.org/10.5061/dryad. $\operatorname{t} 4 \mathrm{r} \ln 64) .{ }^{31}$ 


\section{Statistical analysis}

We determined the 10-year risk of follow-up coronary events (defined as MI or sudden cardiac deaths), recurrent ischemic stroke, major ischemic vascular events (defined as coronary event or recurrent ischemic stroke, whichever happens first), and major bleeds (extracranial and intracerebral hemorrhage) from Kaplan-Meier analyses, stratified by history of coexisting cardiovascular disease, and assessed any difference with logrank test. We used similar analyses for the 10-year risk stratified by stroke subtype. We also compared risks of follow-up events between patients with and without coexisting cardiovascular disease using logistic regression analysis adjusted for age and sex. All analyses were done using $\mathrm{R}$ version 3.1.3.

\section{Data availability}

Requests for anonymized data will be considered by the corresponding author.

\section{Results}

Of 2,555 patients (949 TIA and 1,606 ischemic stroke; mean age 74 years; $21 \%$ aged $\geq 85$ years), $443(17.3 \%)$ had a previous TIA or stroke before their index event and 640 (25.0\%) had history of coexisting stable cardiovascular disease (449 coronary artery disease only; 103 peripheral artery disease only; 88 both [tables 1 and 2]). At baseline, the mean total cholesterol level was $5.05( \pm 1.27) \mathrm{mmol} / \mathrm{L}$, ranging from 4.86 $( \pm 1.30) \mathrm{mmol} / \mathrm{L}$ in patients on statin treatment prior to the TIA/stroke to $5.12( \pm 1.25) \mathrm{mmol} / \mathrm{L}$ in those with no statin $(p$ $<0.001)$.

Patients with coexisting cardiovascular disease were older (mean age 78 vs 73 years, $p<0.001$ ), had more atherosclerotic risk factors, and were more likely to be on antithrombotics, antihypertensives, and statins before the TIA/ stroke than those without (table 1 and table e-2, doi.org/10. 5061/dryad.t4r $\ln 64$ ). Rates of use of secondary preventive therapies were high at 1 -year follow-up, with similar rates of use of antithrombotics and statins in patients with and without coexisting cardiovascular disease, but rate of use of antihypertensives remained higher in those with coexisting cardiovascular disease (table 1 and table e-2, doi.org/10. 5061/dryad.t4r1n64). Patients with TIA/stroke due to large artery disease (TOAST classification) and cardioembolism had the highest prevalence of coexisting cardiovascular disease $(p<0.001$, table 2$)$.

During 9,148 patient-years of follow-up, there were 158 coronary events (29 STEMI; 89 N-STEMI; 40 sudden cardiac deaths), 413 recurrent ischemic strokes, and 1,118 deaths. Patients with coexisting cardiovascular disease comprised $25 \%$ of all patients but accounted for $51 \%$ of coronary events and $28 \%$ of recurrent ischemic stroke during follow-up. In patients with noncardioembolic TIA/ischemic stroke and after exclusion of those on anticoagulants, 82 extracranial bleeds and 20 intracerebral hemorrhages occurred.
The 10-year risk of coronary events (10.6\%, $95 \%$ confidence interval 8.8-12.4 overall) was higher in patients with coexisting cardiovascular disease than in those without $(22.8 \%$, 17.4-27.9; vs 7.1\%, 5.3-8.8; $p<0.001$; age- and sex-adjusted hazard ratio [HR] 3.07, 2.24-4.21, table 3, figure 1 , and figures e-1 and e-2, doi.org/10.5061/dryad.t4r1n64), particularly in patients aged $<75$ years (16.69\%, 9.65-23.19; vs $3.78 \%$, 2.09-5.44; $p<0.001 ;$ age-and sex-adjusted HR 4.33, 2.42-7.73, table 3).

The 10-year risk of recurrent ischemic stroke $(25.3 \%$, 22.6-27.8 overall) was also higher in patients with coexisting cardiovascular disease than those without $(31.5 \%$, $25.1-37.4$; vs $23.4 \%, 20.5-26.3$; $p=0.0049$; age- and sexadjusted HR 1.23, 0.99-1.53, table 3 , figure 1 , and figure e-3, doi.org/10.5061/dryad.t4r1n64), particularly in patients aged $<75$ years $(30.3 \%, 21.4-38.3$; vs $17.6 \%$, 14.3-20.7; $p<0.001$; adjusted HR 1.74, 1.23-2.46, table 3). Patients with coexisting cardiovascular disease also had a higher 10-year risk of all major ischemic vascular events than those without $(45.1 \%, 38.4-51.1$; vs $28.7 \%, 25.3-31.9$; $p<0.001$; age- and sex-adjusted HR 1.56, 1.30-1.88, table 3 , figure 1).

In patients with noncardioembolic TIA/stroke and after exclusion of those on anticoagulants, the 10-year risk of major extracranial bleeds was higher in those with coexisting cardiovascular disease (table 3 ), particularly in patients aged $<75$ years $(8.1 \%, 2.8-13.0$; vs $3.5 \%, 1.6-5.3 ; p=0.0050$; age- and sex-adjusted HR 2.71, 1.16-6.30). The excess risk of major extracranial bleeds in those with coexisting cardiovascular disease was not limited only to the very early follow-up period (figure 1). In contrast, patients with coexisting cardiovascular disease were not at increased risk of intracerebral hemorrhage (1.5\%, $0-4.3$; vs $2.5 \%, 1.0-4.0 ; p=0.09$; age- and sex-adjusted HR $0.17,0.02-1.26$, table 3 ).

The subgroup of patients with (TOAST) large artery disease had the highest 10-year risk of coronary events compared to the other stroke subtypes $(16.1 \%, 10.3-21.6, p<$ 0.001 ) and among the highest 10 -year risk of recurrent ischemic stroke $(26.3 \%, 18.9-33.1, p<0.001$, table 4, figure 2 ). However, in patients with (TOAST) large artery disease, risk of coronary events remained increased in those with coexisting cardiovascular disease compared to those without (10-year risk 33.5\%, 15.9-47.4; vs 9.5\%, 4.2-14.6; $p<0.001$; age- and sex-adjusted HR 3.39, 1.65-6.96) while risk of recurrent ischemic stroke was high irrespective of presence of coexisting cardiovascular disease (10-year risk $26.4 \%, 12.6-38.1$; vs $26.3 \%, 17.3-34.4 ; p=0.79$; age- and sex-adjusted HR 1.02, 0.57-1.83, figure 3 and table e-3, doi. org/10.5061/dryad.t4r1n64). Patients with (TOAST) large artery disease also had a high 10 -year risk of major extracranial bleeds $(11.1 \%, 1.8-19.5, p=0.85)$ but a relatively low risk of intracerebral hemorrhage $(0.4 \%, 0.0-1.1$, $p=0.26$, table 4 and table e-3, doi.org/10.5061/dryad. t4r $\ln 64)$. In contrast, the subgroup of patients with 
Table 1 Baseline characteristics in patients with TIA or ischemic stroke, stratified by history of coexisting cardiovascular disease

\begin{tabular}{|c|c|c|c|c|}
\hline \multirow[b]{2}{*}{ Baseline characteristics } & \multirow{2}{*}{$\begin{array}{l}\text { Total } \\
(n=2,555)\end{array}$} & \multicolumn{2}{|c|}{ Coexisting cardiovascular disease } & \multirow[b]{2}{*}{$p$ Value } \\
\hline & & Yes $(n=640)$ & No $(n=1915)$ & \\
\hline \multicolumn{5}{|l|}{ Demographics } \\
\hline Mean age (SD), y & $73.9(13.0)$ & $78.0(10.0)$ & $72.6(13.6)$ & $<0.001$ \\
\hline Age $\geq 75$ years & $1,404(55.0)$ & $444(69.4)$ & $960(50.1)$ & $<0.001$ \\
\hline Men & $1,262(49.4)$ & $354(55.3)$ & $908(47.4)$ & $<0.001$ \\
\hline \multicolumn{5}{|l|}{ Prior comorbidities } \\
\hline Previous TIA & $\begin{array}{l}231 \\
(9.0)\end{array}$ & $93(14.5)$ & $138(7.2)$ & $<0.001$ \\
\hline Previous stroke & $264(10.3)$ & $95(14.8)$ & $169(8.8)$ & $<0.001$ \\
\hline Atrial fibrillation & $522(20.4)$ & 204 (31.9) & $318(16.6)$ & $<0.001$ \\
\hline Hypertension & $1,537(60.1)$ & $492(76.9)$ & $1,045(54.6)$ & $<0.001$ \\
\hline Diabetes mellitus & $357(14.0)$ & $156(24.4)$ & $201(10.5)$ & $<0.001$ \\
\hline Hypercholesterolemia & $782(30.6)$ & $334(52.2)$ & $448(23.4)$ & $<0.001$ \\
\hline History of smoking ${ }^{a}$ & $1,416(55.4)$ & $403(63.0)$ & $1,013(52.9)$ & $<0.001$ \\
\hline Current smoker ${ }^{a}$ & $361(14.1)$ & $64(10.0)$ & $297(15.5)$ & $<0.001$ \\
\hline Family history of premature myocardial infarction ${ }^{b}$ & $224(8.8)$ & $86(16.5)$ & $138(8.9)$ & $<0.001$ \\
\hline Renal insufficiency ${ }^{c}$ & $132(5.2)$ & $56(8.8)$ & $76(4.0)$ & $<0.001$ \\
\hline \multicolumn{5}{|l|}{ TIA/stroke subtypes (TOAST classification) } \\
\hline Large artery disease & $280(11.0)$ & $85(13.3)$ & $195(10.2)$ & 0.03 \\
\hline Cardioembolic & $669(26.2)$ & $226(35.3)$ & $443(23.1)$ & $<0.001$ \\
\hline Small vessel disease & $317(12.4)$ & $48(7.5)$ & $269(14.0)$ & $<0.001$ \\
\hline Cryptogenic & $811(31.7)$ & $140(21.9)$ & $671(35.0)$ & $<0.001$ \\
\hline More than one cause & $90(3.5)$ & $45(7.0)$ & $45(2.3)$ & $<0.001$ \\
\hline Unknown & $331(13.0)$ & $88(13.8)$ & $242(12.6)$ & 0.47 \\
\hline Other causes & $57(2.2)$ & $8(1.3)$ & $49(2.6)$ & 0.05 \\
\hline \multicolumn{5}{|l|}{ Baseline therapies } \\
\hline Antithrombotics & $1,100(43.1)$ & $496(77.5)$ & $603(31.5)$ & $<0.001$ \\
\hline Antihypertensives & $1,486(58.2)$ & $530(82.8)$ & 956 (49.9) & $<0.001$ \\
\hline Statins & $684(26.8)$ & $358(55.9)$ & $326(17.0)$ & $<0.001$ \\
\hline $\begin{array}{l}\text { Secondary prevention medication at 1-month } \\
\text { follow-up }{ }^{d}\end{array}$ & $n=2,339$ & $n=559$ & $n=1,780$ & \\
\hline Antithrombotics & $2,196(93.9)$ & $522(93.4)$ & $1,673(94.0)$ & 0.60 \\
\hline Antihypertensives & $1,771(75.7)$ & $482(86.2)$ & $1,288(72.4)$ & $<0.001$ \\
\hline Statins & $1,755(75.0)$ & $451(80.7)$ & $1,304(73.3)$ & $<0.001$ \\
\hline $\begin{array}{l}\text { Secondary prevention medication at 1-year } \\
\text { follow-up }{ }^{d}\end{array}$ & $\mathrm{n}=1,976$ & $n=458$ & $n=1,518$ & \\
\hline Antithrombotics & $1,884(95.3)$ & $437(95.4)$ & $1,447(95.3)$ & 0.85 \\
\hline Antihypertensives & 1,572 (79.6) & $398(86.9)$ & $1,174(77.3)$ & $<0.001$ \\
\hline
\end{tabular}


Table 1 Baseline characteristics in patients with TIA or ischemic stroke, stratified by history of coexisting cardiovascular disease (continued)

\begin{tabular}{|c|c|c|c|c|}
\hline \multirow[b]{2}{*}{ Baseline characteristics } & \multirow{2}{*}{$\begin{array}{l}\text { Total } \\
(n=2,555)\end{array}$} & \multicolumn{2}{|c|}{ Coexisting cardiovascular disease } & \multirow[b]{2}{*}{$p$ Value } \\
\hline & & Yes $(n=640)$ & No $(n=1915)$ & \\
\hline Statins & $1,589(80.4)$ & $370(80.8)$ & $1,219(80.3)$ & 0.87 \\
\hline
\end{tabular}

Abbreviation: TOAST $=$ Trial of Org 10172 in Acute Stroke Treatment.

Data are $\mathrm{n}(\%)$, unless otherwise specified. $p$ For comparison between patients with and without coexisting cardiovascular disease $\left(\chi^{2}\right.$ test for categorical variables and $t$ test for continuous variable).

${ }^{\text {a }}$ Data missing for 7 patients.

${ }^{\mathrm{b}}$ Data missing for 476 patients.

${ }^{c}$ Data missing for 13 patients.

${ }^{d}$ Patients who died before the follow-up were not included.

cryptogenic stroke had the lowest 10-year risk of recurrent ischemic stroke $(19.5 \%, 15.6-23.3, p<0.001)$ and of coronary events compared to the other stroke subtypes ( $8.3 \%$,
5.4-11.1, table 4, figure 2), but a relatively high 10-year risk of intracerebral hemorrhage $(2.5 \%, 0.76-4.2, p=0.26$, table 4).

Table 2 Baseline characteristics in patients with TIA or ischemic stroke, stratified by TIA/stroke subtypes (Trial of Org 10172 in Acute Stroke Treatment [TOAST] classification)

\begin{tabular}{|c|c|c|c|c|c|}
\hline Baseline characteristics & $\begin{array}{l}\text { Large artery disease } \\
(\mathrm{n}=\mathbf{2 8 0})\end{array}$ & $\begin{array}{l}\text { Cardioembolic } \\
(\mathrm{n}=669)\end{array}$ & $\begin{array}{l}\text { Small vessel disease } \\
(n=317)\end{array}$ & $\begin{array}{l}\text { Cryptogenic } \\
(\mathrm{n}=\mathbf{8 1 1})\end{array}$ & $\begin{array}{l}p \\
\text { Value }\end{array}$ \\
\hline \multicolumn{6}{|l|}{ Demographics } \\
\hline Mean (SD) age, y & $73.3(10.3)$ & 77.9 (11.9) & $69.7(12.6)$ & $70.3(12.8)$ & $<0.001$ \\
\hline Age $\geq 75$ years & $137(48.9)$ & $469(70.1)$ & $121(38.2)$ & $346(42.7)$ & $<0.001$ \\
\hline Men & $172(61.4)$ & $306(45.7)$ & $182(57.4)$ & 402 (49.6) & $<0.001$ \\
\hline \multicolumn{6}{|l|}{ Prior comorbidities } \\
\hline Coexisting cardiovascular disease & $85(30.6)$ & $226(33.8)$ & $48(15.1)$ & $140(17.3)$ & $<0.001$ \\
\hline Hypertension & $198(70.7)$ & $454(67.9)$ & $183(57.7)$ & 419 (51.7) & $<0.001$ \\
\hline Diabetes mellitus & $52(18.6)$ & $81(12.1)$ & $51(16.1)$ & $99(12.2)$ & 0.0172 \\
\hline Hypercholesterolemia & $121(43.2)$ & $205(30.6)$ & $94(29.7)$ & $228(28.1)$ & $<0.001$ \\
\hline History of smoking & $183(65.3)$ & $348(52.0)$ & $197(62.1)$ & $443(54.6)$ & $<0.001$ \\
\hline \multicolumn{6}{|l|}{ Baseline therapies } \\
\hline Antithrombotics & $106(37.9)$ & $427(63.8)$ & 101 (31.9) & $273(33.7)$ & $<0.001$ \\
\hline Antihypertensives & $187(66.8)$ & $482(72.0)$ & $151(47.6)$ & $394(48.6)$ & $<0.001$ \\
\hline Statins & $102(36.4)$ & $191(28.6)$ & $70(22.1)$ & $205(25.3)$ & 0.03 \\
\hline $\begin{array}{l}\text { Secondary prevention medication at 1-month } \\
\text { follow-up }^{\mathrm{a}}\end{array}$ & $n=273$ & $\mathrm{n}=556$ & $\mathrm{n}=317$ & $\mathrm{n}=808$ & \\
\hline Antithrombotics & $263(96.3)$ & $520(93.5)$ & $307(96.8)$ & $769(95.2)$ & 0.22 \\
\hline Antihypertensives & $224(82.1)$ & $446(80.2)$ & $258(81.4)$ & 589 (72.9) & $<0.001$ \\
\hline Statins & $232(85.0)$ & $380(68.3)$ & $266(83.9)$ & $649(80.3)$ & $<0.001$ \\
\hline Secondary prevention medication at 1-year follow-up ${ }^{a}$ & $n=247$ & $n=436$ & $n=310$ & $n=765$ & \\
\hline Antithrombotics & $239(96.8)$ & $411(94.3)$ & $292(94.2)$ & $719(94.0)$ & 0.53 \\
\hline Antihypertensives & $213(86.2)$ & $359(82.3)$ & $246(79.4)$ & $573(74.9)$ & $<0.001$ \\
\hline Statins & 221 (78.9) & $315(72.2)$ & $256(82.6)$ & $627(82.0)$ & 0.0011 \\
\hline
\end{tabular}

Data are number (\%), unless otherwise specified. $p$ For comparison among all stroke subtypes $\left(\chi^{2}\right.$ test for categorical variables and one-way analysis of variance test for continuous variable).

a Patients who died before the follow-up were not included. 
Table 3 Ten-year risk of coronary events, recurrent ischemic stroke, major ischemic vascular events, intracerebral hemorrhage, and major extracranial bleeds after TIA/ischemic stroke in patients with vs without coexisting cardiovascular disease, stratified by age

\begin{tabular}{|c|c|c|c|c|c|c|c|c|}
\hline \multirow{2}{*}{$\begin{array}{l}\text { Coexisting } \\
\text { cardiovascular disease }\end{array}$} & \multicolumn{2}{|c|}{ Events, $n / N$} & \multicolumn{2}{|c|}{ 10-year risk, \% $(95 \% \mathrm{Cl})$} & \multirow{2}{*}{$\begin{array}{l}p \\
\text { Value }\end{array}$} & \multirow{2}{*}{$\begin{array}{l}\text { Crude HR } \\
(95 \% \mathrm{Cl})\end{array}$} & \multirow{2}{*}{$\begin{array}{l}\text { Age- and sex-adjusted HR } \\
(95 \% \mathrm{Cl})\end{array}$} & \multirow{2}{*}{$\begin{array}{l}p \\
\text { Value }\end{array}$} \\
\hline & Yes & No & Yes & No & & & & \\
\hline \multicolumn{9}{|l|}{ All patients } \\
\hline Coronary events & $\begin{array}{l}80 / \\
640\end{array}$ & $\begin{array}{l}78 / \\
1,915\end{array}$ & $\begin{array}{l}22.83 \\
(17.40-27.91)\end{array}$ & $\begin{array}{l}7.06 \\
(5.30-8.79)\end{array}$ & $<0.001$ & $\begin{array}{l}3.83 \\
(2.81-5.21)\end{array}$ & $3.07(2.24-4.21)$ & $<0.001$ \\
\hline $\begin{array}{l}\text { Recurrent ischemic } \\
\text { stroke }\end{array}$ & $\begin{array}{l}118 / \\
640\end{array}$ & $\begin{array}{l}295 / \\
1,915\end{array}$ & $\begin{array}{l}31.51 \\
(25.10-37.37)\end{array}$ & $\begin{array}{l}23.43 \\
(20.49-26.25)\end{array}$ & 0.0049 & $\begin{array}{l}1.36 \\
(1.10-1.68)\end{array}$ & $1.23(0.99-1.53)$ & 0.06 \\
\hline $\begin{array}{l}\text { Major ischemic } \\
\text { vascular events }\end{array}$ & $\begin{array}{l}175 / \\
640\end{array}$ & $\begin{array}{l}345 / \\
640\end{array}$ & $\begin{array}{l}45.12 \\
(38.44-51.07)\end{array}$ & $\begin{array}{l}28.65 \\
(25.28-31.86)\end{array}$ & $<0.001$ & $\begin{array}{l}1.76 \\
(1.47-2.11)\end{array}$ & $1.56(1.30-1.88)$ & $<0.001$ \\
\hline $\begin{array}{l}\text { Intracerebral } \\
\text { hemorrhage }^{a}\end{array}$ & $1 / 414$ & $\begin{array}{l}19 / \\
1,472\end{array}$ & $\begin{array}{l}1.47 \\
(0.00-4.29)\end{array}$ & $\begin{array}{l}2.49 \\
(0.97-3.99)\end{array}$ & 0.09 & $\begin{array}{l}0.21 \\
(0.03-1.54)\end{array}$ & $0.17(0.02-1.26)$ & 0.08 \\
\hline $\begin{array}{l}\text { Major extracranial } \\
\text { bleeds }^{\mathrm{a}}\end{array}$ & $\begin{array}{l}27 / \\
351\end{array}$ & $\begin{array}{l}55 / \\
1,226\end{array}$ & $\begin{array}{l}11.14 \\
(6.56-15.50)\end{array}$ & $\begin{array}{l}7.44 \\
(5.09-9.74)\end{array}$ & 0.0050 & $\begin{array}{l}1.91 \\
(1.21-3.03)\end{array}$ & $1.58(0.99-2.52)$ & 0.06 \\
\hline \multicolumn{9}{|l|}{ Age $<75$ years } \\
\hline Coronary events & $\begin{array}{l}23 / \\
196\end{array}$ & $23 / 955$ & $\begin{array}{l}16.69 \\
(9.65-23.19)\end{array}$ & $\begin{array}{l}3.78 \\
(2.09-5.44)\end{array}$ & $<0.001$ & $\begin{array}{l}5.38 \\
(3.06-9.48)\end{array}$ & $4.33(2.42-7.73)$ & $<0.001$ \\
\hline $\begin{array}{l}\text { Recurrent ischemic } \\
\text { stroke }\end{array}$ & $\begin{array}{l}44 / \\
196\end{array}$ & $\begin{array}{l}127 / \\
955\end{array}$ & $\begin{array}{l}30.34 \\
(21.43-38.25)\end{array}$ & $\begin{array}{l}17.61 \\
(14.34-20.74)\end{array}$ & $<0.001$ & $\begin{array}{l}1.87 \\
(1.33-2.62)\end{array}$ & $1.74(1.23-2.46)$ & 0.0012 \\
\hline $\begin{array}{l}\text { Major ischemic } \\
\text { vascular events }\end{array}$ & $\begin{array}{l}59 / \\
196\end{array}$ & $\begin{array}{l}141 / \\
955\end{array}$ & $\begin{array}{l}39.68 \\
(30.39-47.73)\end{array}$ & $\begin{array}{l}20.96 \\
(17.11-24.62)\end{array}$ & $<0.001$ & $\begin{array}{l}2.28 \\
(1.69-3.09)\end{array}$ & $2.10(1.54-2.87)$ & $<0.001$ \\
\hline $\begin{array}{l}\text { Intracerebral } \\
\text { hemorrhage }^{a}\end{array}$ & $1 / 150$ & $8 / 801$ & $\begin{array}{l}2.27 \\
(0.00-6.58)\end{array}$ & $\begin{array}{l}2.23 \\
(0.31-4.12)\end{array}$ & 0.66 & $\begin{array}{l}0.63 \\
(0.08-5.03)\end{array}$ & $0.36(0.04-2.99)$ & 0.41 \\
\hline $\begin{array}{l}\text { Major extracranial } \\
\text { bleeds }^{\mathrm{a}}\end{array}$ & $9 / 126$ & $16 / 653$ & $\begin{array}{l}8.07 \\
(2.81-13.04)\end{array}$ & $\begin{array}{l}3.45 \\
(1.61-5.26)\end{array}$ & 0.0050 & $\begin{array}{l}3.04 \\
(1.34-6.88)\end{array}$ & $2.71(1.16-6.30)$ & 0.0209 \\
\hline \multicolumn{9}{|l|}{ Age $\geq 75$ years } \\
\hline Coronary events & $\begin{array}{l}57 / \\
444\end{array}$ & $55 / 960$ & $\begin{array}{l}27.99 \\
(19.05-35.94)\end{array}$ & $\begin{array}{l}12.44 \\
(8.38-16.31)\end{array}$ & $<0.001$ & $\begin{array}{l}2.64 \\
(1.83-3.82)\end{array}$ & $2.59(1.79-3.77)$ & $<0.001$ \\
\hline $\begin{array}{l}\text { Recurrent ischemic } \\
\text { stroke }\end{array}$ & $\begin{array}{l}74 / \\
444\end{array}$ & $\begin{array}{l}168 / \\
960\end{array}$ & $\begin{array}{l}33.81 \\
(23.47-42.76)\end{array}$ & $\begin{array}{l}32.19 \\
(26.42-37.51)\end{array}$ & 0.89 & $\begin{array}{l}1.02 \\
(0.78-1.34)\end{array}$ & $1.05(0.80-1.38)$ & 0.79 \\
\hline $\begin{array}{l}\text { Major ischemic } \\
\text { vascular events }\end{array}$ & $\begin{array}{l}116 / \\
444\end{array}$ & $\begin{array}{l}204 / \\
960\end{array}$ & $\begin{array}{l}50.66 \\
(39.87-59.51)\end{array}$ & $\begin{array}{l}39.69 \\
(33.37-45.41)\end{array}$ & 0.0096 & $\begin{array}{l}1.35 \\
(1.08-1.70)\end{array}$ & $1.37(1.09-1.73)$ & 0.0063 \\
\hline $\begin{array}{l}\text { Intracerebral } \\
\text { hemorrhage }^{a}\end{array}$ & $0 / 264$ & $11 / 671$ & 0 & $\begin{array}{l}2.49 \\
(0.76-4.20)\end{array}$ & 0.0443 & $\begin{array}{l}0.01 \\
(0.00-100)\end{array}$ & $0.01(0.00-100)$ & 0.59 \\
\hline $\begin{array}{l}\text { Major extracranial } \\
\text { bleeds }\end{array}$ & $\begin{array}{l}18 / \\
225\end{array}$ & $39 / 573$ & $\begin{array}{l}19.21 \\
(3.88-32.10)\end{array}$ & $\begin{array}{l}14.15 \\
(8.01-19.87)\end{array}$ & 0.34 & $\begin{array}{l}1.31 \\
(0.75-2.29)\end{array}$ & $1.28(0.73-2.56)$ & 0.39 \\
\hline
\end{tabular}

Abbreviations: $\mathrm{Cl}$ = confidence interval; $\mathrm{HR}=$ hazard ratio.

Major ischemic vascular event was a component of coronary event or recurrent ischemic stroke. Information on coronary events, recurrent ischemic stroke, and intracerebral hemorrhage was available in cases recruited from April 1, 2002, to March 31, 2014, with follow-up until September 30, 2014. Information on major extracranial bleeds was available in cases recruited from April 1, 2002, to March 31, 2012, with follow-up until March 31, 2013.

a Patients with cardioembolic TIA/ischemic stroke and those on anticoagulants were excluded.

\section{Discussion}

In this population-based cohort of consecutive TIA/stroke patients on current standard management, those with coexisting cardiovascular disease were at significantly higher 10 -year risk of coronary events and recurrent ischemic stroke than those without, particularly at age $<75$ years ( $16.7 \%$ vs $3.8 \%$ and $30.3 \%$ vs $17.6 \%$, respectively). However, in younger patients with noncardioembolic TIA/ stroke (i.e., on antiplatelet therapy for secondary prevention), 10 -year risk of major extracranial bleeds was also higher in those with coexisting cardiovascular disease ( $8.1 \%$ vs $3.5 \%$ ), although there was no increased risk of intracerebral hemorrhage. More intensive lipid-lowering therefore might be justified in patients with coexisting cardiovascular disease, although economic evaluation of PCSK9 inhibitors in addition to statin therapy in similar patient populations suggests that treatment might still exceed the generally accepted cost-effectiveness 
Figure 1 Risks of coronary events, recurrent ischemic stroke, major ischemic vascular events, and major bleeds after TIA or ischemic stroke, stratified by history of coexisting cardiovascular disease
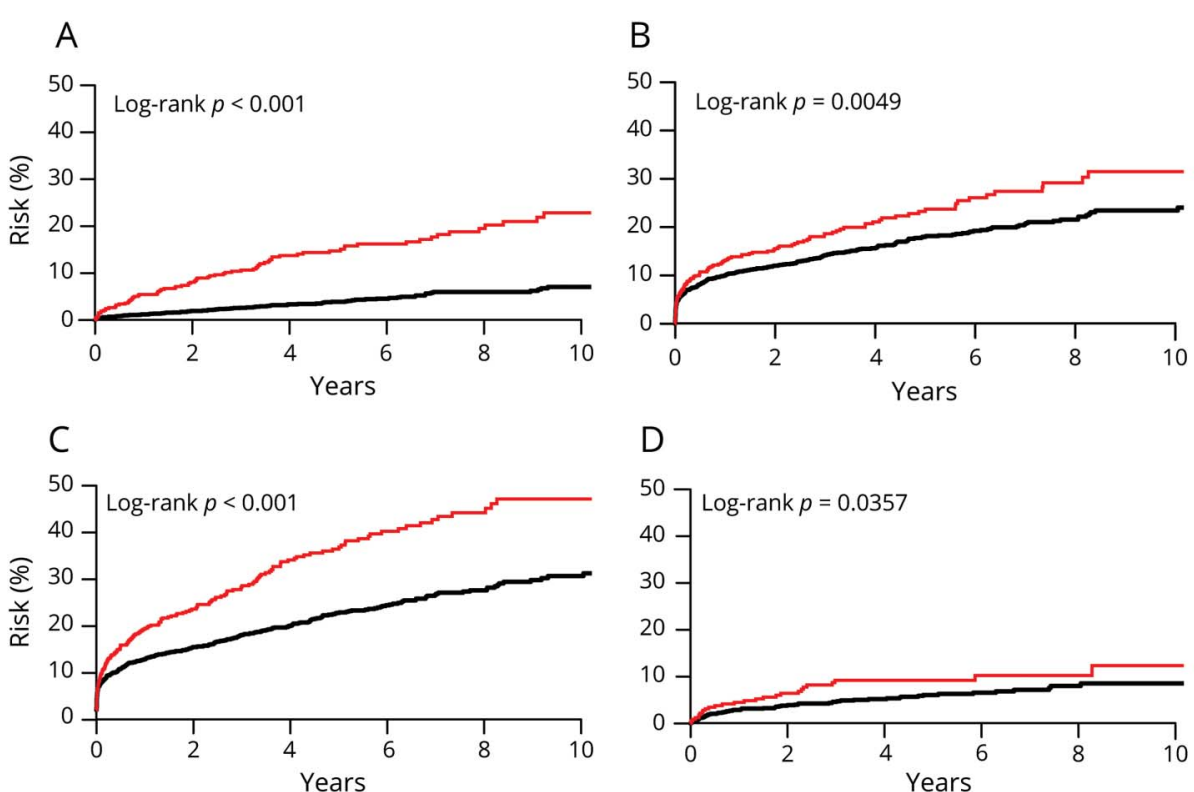

$\mathrm{D}$
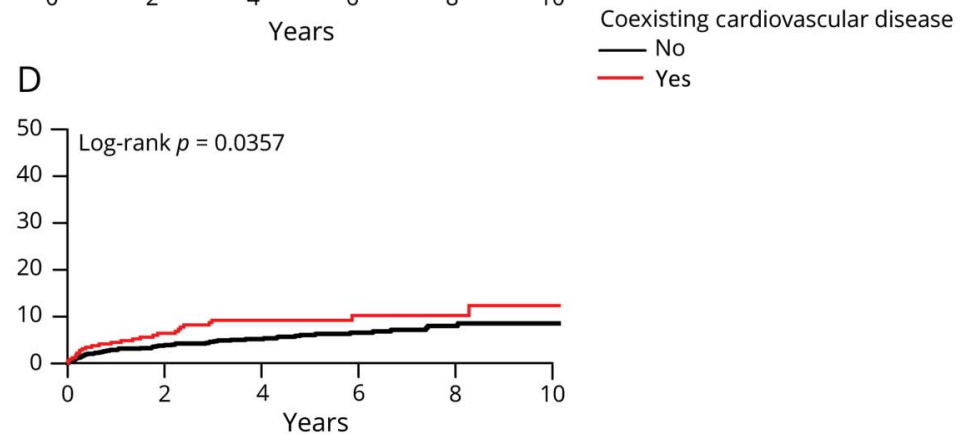

(A) Coronary events. (B) Recurrent ischemic stroke. (C) Major ischemic vascular events. (D) Major bleeds. Major ischemic vascular event was a component of coronary event or recurrent ischemic stroke. Risk of major bleeds was calculated in noncardioembolic patients with TIA or ischemic stroke and after exclusion of those on anticoagulants.

threshold, ${ }^{32}$ and there is probably still potential for more intensive treatment with statins and other lipid-lowering drugs. Benefit from increased antithrombotic treatment might be offset by the higher risk of extracranial bleeding, particularly in older patients, in whom bleeds are more likely to be disabling or fatal. $^{33}$
We found that the subgroup of patients with large artery disease (TOAST classification) were at high risk of ischemic events, consistent with previous findings. ${ }^{34,35}$ However, the current absolute risk of ischemic events (10-year risk of coronary events of $16.1 \%$ and 10 -year risk of recurrent ischemic stroke of $26.3 \%)$ was lower than previously reported (10-year

Table 4 Ten-year risk of coronary events, recurrent ischemic stroke, major ischemic vascular events, intracerebral hemorrhage, and major extracranial bleeds after TIA or ischemic stroke, stratified by stroke subtype

\begin{tabular}{|c|c|c|c|c|c|c|c|c|c|}
\hline & \multicolumn{2}{|c|}{$\begin{array}{l}\text { Large artery disease } \\
(n=280)\end{array}$} & \multicolumn{2}{|c|}{ Cardioembolic $(n=669)$} & \multicolumn{2}{|c|}{$\begin{array}{l}\text { Small vessel disease } \\
(\mathrm{n}=317)\end{array}$} & \multicolumn{2}{|c|}{ Cryptogenic $(n=811)$} & \multirow{2}{*}{$\begin{array}{l}p \\
\text { Value }\end{array}$} \\
\hline & $\mathbf{n}$ & $\%(95 \% \mathrm{Cl})$ & $\mathbf{n}$ & $\%(95 \% \mathrm{Cl})$ & $\mathbf{n}$ & $\%(95 \% \mathrm{Cl})$ & $\mathbf{n}$ & $\%(95 \% \mathrm{Cl})$ & \\
\hline Coronary events & 40 & $\begin{array}{l}16.13 \\
(10.28-21.60)\end{array}$ & 37 & $\begin{array}{l}11.19 \\
(6.91-15.27)\end{array}$ & 19 & $7.87(4.22-11.38)$ & 41 & $8.29(5.38-11.11)$ & $<0.001$ \\
\hline Recurrent ischemic stroke & 54 & $\begin{array}{l}26.34 \\
(18.89-33.11)\end{array}$ & 114 & $\begin{array}{l}31.91 \\
(24.76-38.38)\end{array}$ & 64 & $\begin{array}{l}25.91 \\
(19.47-31.84)\end{array}$ & 111 & $\begin{array}{l}19.50 \\
(15.57-23.25)\end{array}$ & $<0.001$ \\
\hline $\begin{array}{l}\text { Major ischemic vascular } \\
\text { events }\end{array}$ & 71 & $\begin{array}{l}36.73 \\
(28.18-44.26)\end{array}$ & 140 & $\begin{array}{l}39.02 \\
(31.35-45.84)\end{array}$ & 78 & $\begin{array}{l}31.58 \\
(24.82-37.74)\end{array}$ & 139 & $\begin{array}{l}25.70 \\
(20.87-30.23)\end{array}$ & $<0.001$ \\
\hline Intracerebral hemorrhage ${ }^{a}$ & 1 & $0.37(0.00-1.09)$ & NA & NA & 2 & $1.90(0.00-4.85)$ & 11 & $2.50(0.76-4.21)$ & 0.26 \\
\hline Major extracranial bleeds ${ }^{a}$ & 11 & $\begin{array}{l}11.09 \\
(1.84-19.48)\end{array}$ & NA & NA & 16 & $7.65(3.57-11.56)$ & 31 & $7.31(4.27-10.27)$ & 0.85 \\
\hline
\end{tabular}

Abbreviation: $\mathrm{Cl}=$ confidence interval.

$p$ Values are log-rank values. Major ischemic vascular event was a composite of recurrent ischemic stroke or coronary event. Information on coronary events, recurrent ischemic stroke, and intracerebral hemorrhage was available in cases recruited from April 1, 2002, to March 31, 2014, with follow-up until September 30, 2014. Information on major extracranial bleeds was available in cases recruited from April 1, 2002, to March 31, 2012, with follow-up until March 31, 2013, including 235 patients with large artery disease, 277 with small vessel disease, and 669 with cryptogenic TIA/stroke.

a Patients with cardioembolic TIA/ischemic stroke and those on anticoagulants were excluded. 
Figure 2 Risks of coronary events and recurrent ischemic stroke after TIA or ischemic stroke, stratified by stroke subtype (Trial of Org 10172 in Acute Stroke Treatment [TOAST] classification)

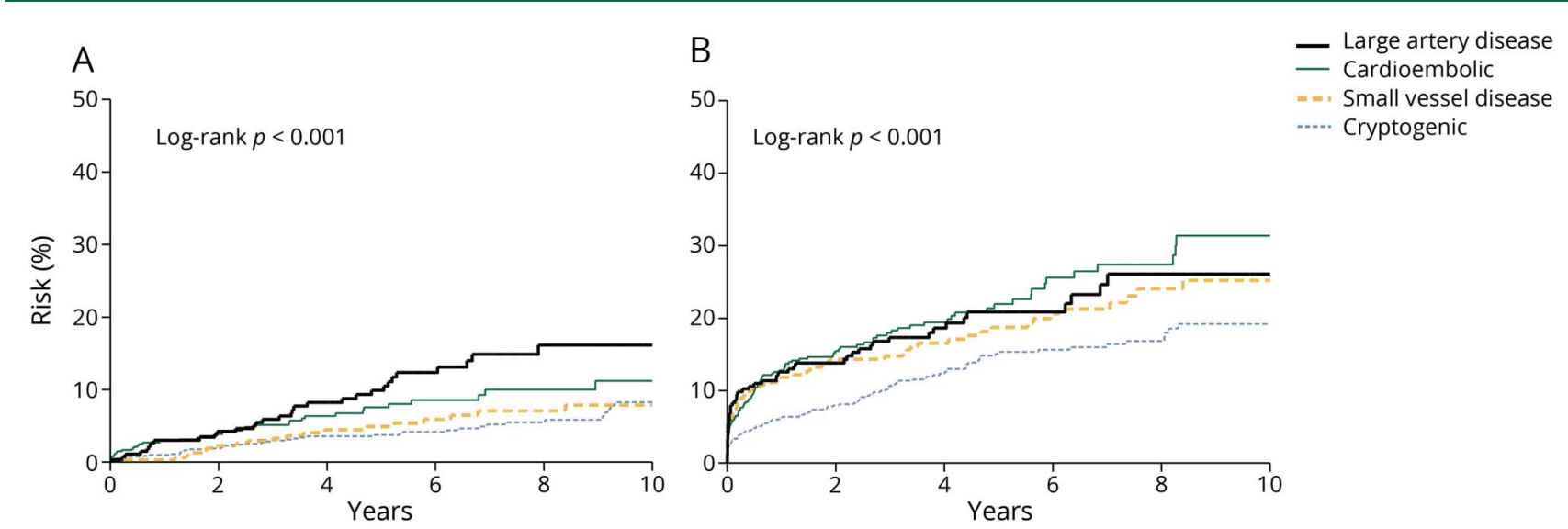

(A) Coronary events. (B) Recurrent ischemic stroke.

risk $>20 \%$ and $>30 \%$, respectively). ${ }^{34,35}$ We also found that stratification by presence of coexisting cardiovascular disease identified individuals at higher risk of coronary events, with 10-year risk ranging from $9.5 \%$ in patients without coexisting cardiovascular disease to $33.5 \%$ in those with. The suggestion that carotid stenosis should be considered as a coronary heart disease equivalent (defined as 10 -year risk $\geq 20 \%^{36}$ ) ${ }^{37}$ was based mainly on data from trials of endarterectomy performed in the 1980s and early 1990s, which reported risks of coronary events of more than $20 \%$ over 10 years. ${ }^{34,35}$ The risk of MI after TIA/stroke has declined over time, ${ }^{2}$ but only one recent study reported the risk in patients with TIA/stroke due to large artery disease, with an annual risk of $0.8 \%,{ }^{38}$ although prevalence of coronary artery disease was lower $(11 \%)$ than in our cohort (30\%) and mean follow-up was only 2.1 years. We found that most coronary events occurred in the subset of patients with large artery disease who had prior coexisting cardiovascular disease.

Figure 3 Risks of coronary events, recurrent ischemic stroke, major ischemic vascular events, and major bleeds after TIA or ischemic stroke due to large artery disease (Trial of Org 10172 in Acute Stroke Treatment [TOAST] classification), stratified by history of coexisting cardiovascular disease

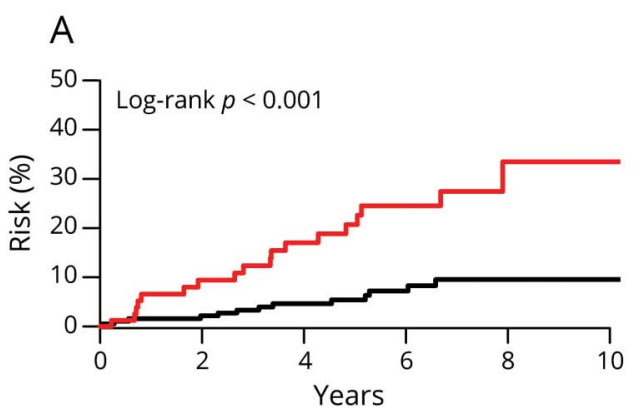

C

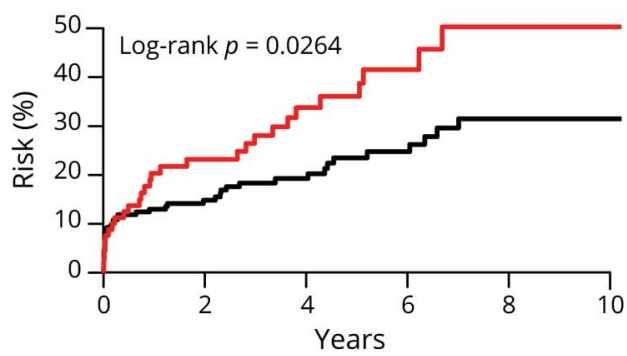

B

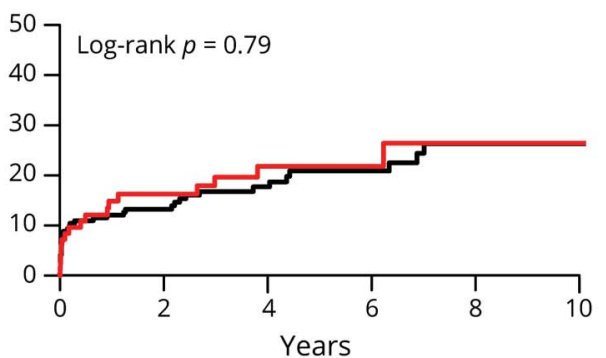

D

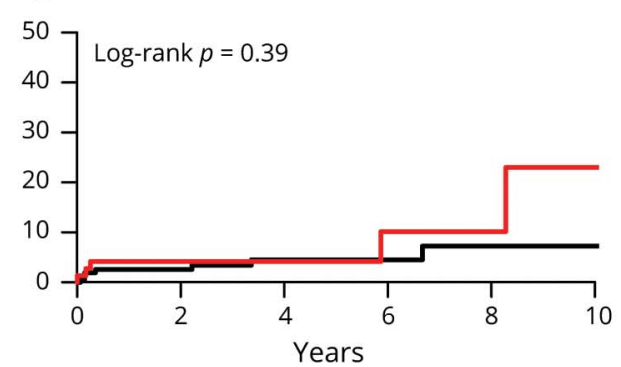

(A) Coronary events. (B) Recurrent ischemic stroke. (C) Major ischemic vascular events. (D) Major bleeds. Major ischemic vascular event was a component of coronary event or recurrent ischemic stroke. Risk of major bleeds was calculated in noncardioembolic patients with TIA or ischemic stroke and after exclusion of those on anticoagulants. 
We also found that patients with cryptogenic TIA/stroke, in whom the prevalence of coexisting cardiovascular disease was relatively low, had the lowest risk of coronary events or recurrent ischemic stroke, but a relatively high risk of intracerebral hemorrhage compared to the other noncardioembolic subtypes. Patients with cryptogenic TIA/ stroke were younger than those with other subtypes and had less hypertension and diabetes, which are known risk factors for intracerebral hemorrhage, ${ }^{21,39}$ and their higher risk of intracerebral hemorrhage remains difficult to explain. Notwithstanding the results of ongoing trials of anticoagulation vs antiplatelet treatment in patients with cryptogenic stroke, ${ }^{40}$ future trials on more intensive antithrombotic management might focus on subgroups of patients with TIA/stroke with a high risk of recurrent ischemic events and a lower risk of bleeding.

Hypertension is a strong predictor of intracerebral hemorrhage ${ }^{21}$ and blood pressure management in patients with TIA/stroke significantly reduces the risk of intracerebral hemorrhage, with benefit increasing over time. ${ }^{41}$ The prevalence of history of hypertension was higher in patients with coexisting cardiovascular disease than in those without and the rates of use of antihypertensives at both baseline and 1 year were also higher, which might at least partly explain the relatively low risk of intracerebral hemorrhage in patients with coexisting cardiovascular disease.

Although we consider our findings to be valid, our study has limitations. First, our population consisted of a predominantly white British population, which will limit generalizability. Second, rates of use of secondary prevention medication were high, but were not $100 \%$. However, our population-based cohort includes many frail and very elderly patients, often with dementia, prior gastrointestinal bleeding, or terminal illness, and some patients refuse medication for other reasons. Nevertheless, rates of medication use at 1 month and 1 year were higher than in the majority of other similar cohorts, ${ }^{42-45}$ and can be regarded as being based closely on current guidelines for secondary prevention. Third, although all hospital admissions for chest pain were reviewed, details were only collected for MI and we did not report information on angina pectoris. Indeed, data on angina are considered to be less reliable than those on MI because underdiagnosed angina is estimated to be common and not all patients with angina symptoms seek medical attention. ${ }^{46}$ Finally, diagnosis of MI has evolved over the last 2 decades $^{29,47}$ and so our findings may not be fully generalizable to future clinical practice. Similarly, those centers that use a DWI-based definition of TIA vs ischemic stroke may obtain higher estimate of recurrent stroke risk.

Patients with TIA/ischemic stroke with coexisting cardiovascular disease have a high risk of recurrent ischemic events on current standard management. More intensive lipid-lowering therefore might be justified, but benefit from increased antithrombotic treatment might be offset by the higher risk of extracranial bleeding, particularly in older patients.

\section{Acknowledgment}

The authors thank the staff in the general practices that collaborated in OXVASC: Abingdon Surgery; Malthouse Surgery, Abingdon; Marcham Road Family Health Centre, Abingdon; The Health Centre, Berinsfield; Key Medical Practice, Kidlington, Oxford; East Oxford Health Centre; and Church Street Practice, Wantage. The authors acknowledge the use of the facilities of the Acute Vascular Imaging Centre, Oxford, UK.

\section{Study funding}

OXVASC is funded by the National Institute for Health Research (NIHR), Oxford Biomedical Research Centre, Wellcome Trust, Wolfson Foundation, and the British Heart Foundation. Professor Rothwell is in receipt of an NIHR Senior Investigator award. Dr. Boulanger was funded by SFNV-France AVC, Société Francaise de Neurologie-JNLF, Fondation Planiol pour l'Etude du Cerveau, and l'Institut Servier.

\section{Disclosure}

The authors report no disclosures relevant to the manuscript. Go to Neurology.org/N for full disclosures.

\section{Publication history}

Received by Neurology October 4, 2018. Accepted in final form March 21, 2019.

\begin{tabular}{|c|c|c|c|}
\hline Name & Location & Role & Contribution \\
\hline $\begin{array}{l}\text { Marion } \\
\text { Boulanger, } \\
\text { MD }\end{array}$ & $\begin{array}{l}\text { Centre for Prevention } \\
\text { of Stroke and } \\
\text { Dementia, University } \\
\text { of Oxford, UK; } \\
\text { Normandie } \\
\text { Université, UNICAEN, } \\
\text { INSERM U1237, Caen, } \\
\text { France }\end{array}$ & Author & $\begin{array}{l}\text { Collected and } \\
\text { analyzed the data, } \\
\text { interpreted the } \\
\text { results, drafted the } \\
\text { manuscript for } \\
\text { intellectual content }\end{array}$ \\
\hline $\begin{array}{l}\text { Linxin Li, } \\
\text { MD, DPhil }\end{array}$ & $\begin{array}{l}\text { Centre for Prevention } \\
\text { of Stroke and } \\
\text { Dementia, } \\
\text { University of } \\
\text { Oxford, UK }\end{array}$ & Author & $\begin{array}{l}\text { Senior research } \\
\text { fellow in the OXVASC } \\
\text { study; collected the } \\
\text { data }\end{array}$ \\
\hline $\begin{array}{l}\text { Shane } \\
\text { Lyons, MD }\end{array}$ & $\begin{array}{l}\text { Centre for Prevention } \\
\text { of Stroke and } \\
\text { Dementia, } \\
\text { University of } \\
\text { Oxford, UK }\end{array}$ & Author & Collected the data \\
\hline $\begin{array}{l}\text { Nicola G. } \\
\text { Lovett, } \\
\text { MBBS }\end{array}$ & $\begin{array}{l}\text { Centre for Prevention } \\
\text { of Stroke and } \\
\text { Dementia, University } \\
\text { of Oxford, UK }\end{array}$ & Author & Collected the data \\
\hline $\begin{array}{l}\text { Magdalena } \\
\text { M. Kubiak, } \\
\text { MBBS, } \\
\text { MRCP, MD }\end{array}$ & $\begin{array}{l}\text { Centre for Prevention } \\
\text { of Stroke and } \\
\text { Dementia, University } \\
\text { of Oxford, UK }\end{array}$ & Author & Collected the data \\
\hline $\begin{array}{l}\text { Louise } \\
\text { Silver, DPhil }\end{array}$ & $\begin{array}{l}\text { Centre for Prevention } \\
\text { of Stroke and } \\
\text { Dementia, University } \\
\text { of Oxford, UK }\end{array}$ & Author & $\begin{array}{l}\text { Coordinated the } \\
\text { OXVASC study; } \\
\text { collected the data }\end{array}$ \\
\hline
\end{tabular}


Appendix 1 (continued)

\begin{tabular}{llll}
\hline Name & Location & Role & Contribution \\
\hline $\begin{array}{l}\text { Emmanuel } \\
\text { Touzé, MD, } \\
\text { PhD }\end{array}$ & $\begin{array}{l}\text { Normandie } \\
\text { Université, UNICAEN, } \\
\text { INSERM U1237, Caen, } \\
\text { France }\end{array}$ & Author & $\begin{array}{l}\text { Revised the } \\
\text { manuscript for } \\
\text { intellectual content }\end{array}$ \\
\hline $\begin{array}{l}\text { Peter M. } \\
\text { Rothwell, } \\
\text { FMedSci }\end{array}$ & $\begin{array}{l}\text { Centre for Prevention } \\
\text { of Stroke and } \\
\text { Dementia, University } \\
\text { of Oxford, UK }\end{array}$ & Author & $\begin{array}{l}\text { Designed and } \\
\text { conceptualized the } \\
\text { study, interpreted } \\
\text { the data, supervised } \\
\text { funding and revised } \\
\text { the manuscript for } \\
\text { intellectual content, } \\
\text { director of the }\end{array}$ \\
& & OXVASC study
\end{tabular}

Appendix 2 Coinvestigators of the OXVASC study

\begin{tabular}{|c|c|c|c|}
\hline Name & Location & Role & Contribution \\
\hline $\begin{array}{l}\text { Prof. Sarah } \\
\text { Pendlebury, } \\
\text { MD, DPhil }\end{array}$ & $\begin{array}{l}\text { Centre for } \\
\text { Prevention of Stroke } \\
\text { and Dementia, } \\
\text { University of } \\
\text { Oxford, UK }\end{array}$ & $\begin{array}{l}\text { Senior research } \\
\text { fellow }\end{array}$ & $\begin{array}{l}\text { Involved in } \\
\text { the follow-up } \\
\text { of patients }\end{array}$ \\
\hline $\begin{array}{l}\text { Wilhelm } \\
\text { Kuker, MD }\end{array}$ & $\begin{array}{l}\text { Centre for } \\
\text { Prevention of } \\
\text { Stroke and } \\
\text { Dementia, } \\
\text { University of } \\
\text { Oxford, UK }\end{array}$ & Neuroradiologist & $\begin{array}{l}\text { Reviewed } \\
\text { imaging }\end{array}$ \\
\hline $\begin{array}{l}\text { Alastair } \\
\text { Webb, MD, } \\
\text { DPhil }\end{array}$ & $\begin{array}{l}\text { Centre for } \\
\text { Prevention of } \\
\text { Stroke and } \\
\text { Dementia, } \\
\text { University of } \\
\text { Oxford, UK }\end{array}$ & $\begin{array}{l}\text { Senior research } \\
\text { fellow }\end{array}$ & $\begin{array}{l}\text { Involved in } \\
\text { the follow-up } \\
\text { of patients }\end{array}$ \\
\hline $\begin{array}{l}\text { Sara } \\
\text { Mazzucco, } \\
\text { MD, PhD }\end{array}$ & $\begin{array}{l}\text { Centre for } \\
\text { Prevention of } \\
\text { Stroke and } \\
\text { Dementia, } \\
\text { University of } \\
\text { Oxford, UK }\end{array}$ & $\begin{array}{l}\text { Senior research } \\
\text { fellow }\end{array}$ & $\begin{array}{l}\text { Involved in } \\
\text { the follow-up } \\
\text { of patients }\end{array}$ \\
\hline $\begin{array}{l}\text { Gabriel Yiin, } \\
\text { MD, DPhil }\end{array}$ & $\begin{array}{l}\text { Centre for } \\
\text { Prevention of } \\
\text { Stroke and } \\
\text { Dementia, } \\
\text { University of } \\
\text { Oxford, UK }\end{array}$ & $\begin{array}{l}\text { Senior research } \\
\text { fellow }\end{array}$ & $\begin{array}{l}\text { Involved in } \\
\text { the follow-up } \\
\text { of patients }\end{array}$ \\
\hline $\begin{array}{l}\text { Maria Tuna, } \\
\text { MD, DPhil }\end{array}$ & $\begin{array}{l}\text { Centre for } \\
\text { Prevention of } \\
\text { Stroke and } \\
\text { Dementia, } \\
\text { University of } \\
\text { Oxford, UK }\end{array}$ & $\begin{array}{l}\text { Senior research } \\
\text { fellow }\end{array}$ & $\begin{array}{l}\text { Involved in } \\
\text { the follow-up } \\
\text { of patients }\end{array}$ \\
\hline $\begin{array}{l}\text { Dominic } \\
\text { Howard, } \\
\text { MD, DPhil }\end{array}$ & $\begin{array}{l}\text { Centre for } \\
\text { Prevention of } \\
\text { Stroke and } \\
\text { Dementia, } \\
\text { University of } \\
\text { Oxford, UK }\end{array}$ & $\begin{array}{l}\text { Senior research } \\
\text { fellow }\end{array}$ & $\begin{array}{l}\text { Involved in } \\
\text { the follow-up } \\
\text { of patients }\end{array}$ \\
\hline $\begin{array}{l}\text { Ramon } \\
\text { Luengo- } \\
\text { Fernandez, } \\
\text { DPhil }\end{array}$ & $\begin{array}{l}\text { Centre for } \\
\text { Prevention of Stroke } \\
\text { and Dementia, } \\
\text { University of } \\
\text { Oxford, UK }\end{array}$ & $\begin{array}{l}\text { Senior research } \\
\text { fellow }\end{array}$ & $\begin{array}{l}\text { Involved in } \\
\text { the follow-up } \\
\text { of patients }\end{array}$ \\
\hline
\end{tabular}

Appendix 2 (continued)

\begin{tabular}{|c|c|c|c|}
\hline Name & Location & Role & Contribution \\
\hline $\begin{array}{l}\text { Lucy Binney, } \\
\text { MD, DPhil }\end{array}$ & $\begin{array}{l}\text { Centre for } \\
\text { Prevention of } \\
\text { Stroke and } \\
\text { Dementia, } \\
\text { University of } \\
\text { Oxford, UK }\end{array}$ & $\begin{array}{l}\text { Senior research } \\
\text { fellow }\end{array}$ & $\begin{array}{l}\text { Involved in } \\
\text { the follow-up } \\
\text { of patients }\end{array}$ \\
\hline $\begin{array}{l}\text { Ziyah } \\
\text { Mehta, } \\
\text { DPhil }\end{array}$ & $\begin{array}{l}\text { Centre for } \\
\text { Prevention of } \\
\text { Stroke and } \\
\text { Dementia, } \\
\text { University of } \\
\text { Oxford, UK }\end{array}$ & $\begin{array}{l}\text { Senior } \\
\text { statistician }\end{array}$ & $\begin{array}{l}\text { Coordinated } \\
\text { statistical } \\
\text { analyses }\end{array}$ \\
\hline $\begin{array}{l}\text { Sergei } \\
\text { Gutnikov, } \\
\text { DPhil }\end{array}$ & $\begin{array}{l}\text { Centre for } \\
\text { Prevention of } \\
\text { Stroke and } \\
\text { Dementia, } \\
\text { University of } \\
\text { Oxford, UK }\end{array}$ & $\begin{array}{l}\text { Database } \\
\text { manager }\end{array}$ & $\begin{array}{l}\text { Managed the } \\
\text { database of } \\
\text { the study }\end{array}$ \\
\hline Jean Brooks & $\begin{array}{l}\text { Centre for } \\
\text { Prevention of } \\
\text { Stroke and } \\
\text { Dementia, } \\
\text { University of } \\
\text { Oxford, UK }\end{array}$ & $\begin{array}{l}\text { Research } \\
\text { secretary }\end{array}$ & $\begin{array}{l}\text { Involved in } \\
\text { the follow-up } \\
\text { of patients }\end{array}$ \\
\hline $\begin{array}{l}\text { Aubretia } \\
\text { McColl, MD }\end{array}$ & $\begin{array}{l}\text { Centre for } \\
\text { Prevention of } \\
\text { Stroke and } \\
\text { Dementia, } \\
\text { University of } \\
\text { Oxford, UK }\end{array}$ & $\begin{array}{l}\text { Clinical research } \\
\text { fellow }\end{array}$ & $\begin{array}{l}\text { Recruited } \\
\text { patients }\end{array}$ \\
\hline $\begin{array}{l}\text { lain } \\
\text { McGurgan, } \\
\text { MD }\end{array}$ & $\begin{array}{l}\text { Centre for } \\
\text { Prevention of } \\
\text { Stroke and } \\
\text { Dementia, } \\
\text { University of } \\
\text { Oxford, UK }\end{array}$ & $\begin{array}{l}\text { Clinical research } \\
\text { fellow }\end{array}$ & $\begin{array}{l}\text { Recruited } \\
\text { patients }\end{array}$ \\
\hline $\begin{array}{l}\text { Robert } \\
\text { Hurford, MD }\end{array}$ & $\begin{array}{l}\text { Centre for } \\
\text { Prevention of } \\
\text { Stroke and } \\
\text { Dementia, } \\
\text { University of } \\
\text { Oxford, UK }\end{array}$ & $\begin{array}{l}\text { Clinical research } \\
\text { fellow }\end{array}$ & $\begin{array}{l}\text { Recruited } \\
\text { patients }\end{array}$ \\
\hline $\begin{array}{l}\text { Dearbhla } \\
\text { Kelly, MD }\end{array}$ & $\begin{array}{l}\text { Centre for } \\
\text { Prevention of } \\
\text { Stroke and } \\
\text { Dementia, } \\
\text { University of } \\
\text { Oxford, UK }\end{array}$ & $\begin{array}{l}\text { Clinical research } \\
\text { fellow }\end{array}$ & $\begin{array}{l}\text { Recruited } \\
\text { patients }\end{array}$ \\
\hline Sarah Welch & $\begin{array}{l}\text { Centre for } \\
\text { Prevention of } \\
\text { Stroke and } \\
\text { Dementia, } \\
\text { University of } \\
\text { Oxford, UK }\end{array}$ & Research nurse & $\begin{array}{l}\text { Involved in } \\
\text { the follow-up } \\
\text { of patients }\end{array}$ \\
\hline $\begin{array}{l}\text { Ellen } \\
\text { McCulloch }\end{array}$ & $\begin{array}{l}\text { Centre for } \\
\text { Prevention of } \\
\text { Stroke and } \\
\text { Dementia, } \\
\text { University of } \\
\text { Oxford, UK }\end{array}$ & Research nurse & $\begin{array}{l}\text { Involved in } \\
\text { the follow-up } \\
\text { of patients }\end{array}$ \\
\hline Sally Beebe & $\begin{array}{l}\text { Centre for } \\
\text { Prevention of } \\
\text { Stroke and } \\
\text { Dementia, } \\
\text { University of } \\
\text { Oxford, UK }\end{array}$ & Research nurse & $\begin{array}{l}\text { Involved in } \\
\text { the follow-up } \\
\text { of patients }\end{array}$ \\
\hline
\end{tabular}

Continued 
Appendix 2 (continued)

\begin{tabular}{|c|c|c|c|}
\hline Name & Location & Role & Contribution \\
\hline $\begin{array}{l}\text { Karen } \\
\text { Bowsher- } \\
\text { Brown }\end{array}$ & $\begin{array}{l}\text { Centre for } \\
\text { Prevention of } \\
\text { Stroke and } \\
\text { Dementia, } \\
\text { University of } \\
\text { Oxford, UK }\end{array}$ & Research nurse & $\begin{array}{l}\text { Involved in } \\
\text { the follow-up } \\
\text { of patients }\end{array}$ \\
\hline $\begin{array}{l}\text { Josephine } \\
\text { Brooks }\end{array}$ & $\begin{array}{l}\text { Centre for } \\
\text { Prevention of } \\
\text { Stroke and } \\
\text { Dementia, } \\
\text { University of } \\
\text { Oxford, UK }\end{array}$ & Research nurse & $\begin{array}{l}\text { Involved in } \\
\text { the follow-up } \\
\text { of patients }\end{array}$ \\
\hline $\begin{array}{l}\text { Susannah } \\
\text { Rae }\end{array}$ & $\begin{array}{l}\text { Centre for } \\
\text { Prevention of } \\
\text { Stroke and } \\
\text { Dementia, } \\
\text { University of } \\
\text { Oxford, UK }\end{array}$ & Research nurse & $\begin{array}{l}\text { Involved in } \\
\text { the follow-up } \\
\text { of patients }\end{array}$ \\
\hline $\begin{array}{l}\text { Emily-Rose } \\
\text { Vaughan- } \\
\text { Fowler }\end{array}$ & $\begin{array}{l}\text { Centre for } \\
\text { Prevention of } \\
\text { Stroke and } \\
\text { Dementia, } \\
\text { University of } \\
\text { Oxford, UK }\end{array}$ & Research nurse & $\begin{array}{l}\text { Involved in } \\
\text { the follow-up } \\
\text { of patients }\end{array}$ \\
\hline $\begin{array}{l}\text { Robyn } \\
\text { Harris }\end{array}$ & $\begin{array}{l}\text { Centre for } \\
\text { Prevention of } \\
\text { Stroke and } \\
\text { Dementia, } \\
\text { University of } \\
\text { Oxford, UK }\end{array}$ & Research nurse & $\begin{array}{l}\text { Involved in } \\
\text { the follow-up } \\
\text { of patients }\end{array}$ \\
\hline $\begin{array}{l}\text { Anne-Marie } \\
\text { Haigh }\end{array}$ & $\begin{array}{l}\text { Centre for } \\
\text { Prevention of } \\
\text { Stroke and } \\
\text { Dementia, } \\
\text { University of } \\
\text { Oxford, UK }\end{array}$ & Research nurse & $\begin{array}{l}\text { Involved in } \\
\text { the follow-up } \\
\text { of patients }\end{array}$ \\
\hline $\begin{array}{l}\text { Michelle } \\
\text { Wilson }\end{array}$ & $\begin{array}{l}\text { Centre for } \\
\text { Prevention of } \\
\text { Stroke and } \\
\text { Dementia, } \\
\text { University of } \\
\text { Oxford, UK }\end{array}$ & $\begin{array}{l}\text { Research } \\
\text { physiotherapist }\end{array}$ & $\begin{array}{l}\text { Involved in } \\
\text { the follow-up } \\
\text { of patients }\end{array}$ \\
\hline $\begin{array}{l}\text { Fiona } \\
\text { Cuthbertson }\end{array}$ & $\begin{array}{l}\text { Centre for } \\
\text { Prevention of } \\
\text { Stroke and } \\
\text { Dementia, } \\
\text { University of } \\
\text { Oxford, UK }\end{array}$ & $\begin{array}{l}\text { Research } \\
\text { physiotherapist }\end{array}$ & $\begin{array}{l}\text { Involved in } \\
\text { the follow-up } \\
\text { of patients }\end{array}$ \\
\hline Amy Lawson & $\begin{array}{l}\text { Centre for } \\
\text { Prevention of } \\
\text { Stroke and } \\
\text { Dementia, } \\
\text { University of } \\
\text { Oxford, UK }\end{array}$ & $\begin{array}{l}\text { Research } \\
\text { assistant }\end{array}$ & $\begin{array}{l}\text { Involved in } \\
\text { the follow-up } \\
\text { of patients }\end{array}$ \\
\hline $\begin{array}{l}\text { Annette } \\
\text { Burgess, } \\
\text { PhD }\end{array}$ & $\begin{array}{l}\text { Centre for } \\
\text { Prevention of } \\
\text { Stroke and } \\
\text { Dementia, } \\
\text { University of } \\
\text { Oxford, UK }\end{array}$ & $\begin{array}{l}\text { Laboratory } \\
\text { manager }\end{array}$ & $\begin{array}{l}\text { Led the } \\
\text { laboratory } \\
\text { center }\end{array}$ \\
\hline $\begin{array}{l}\text { Deborah } \\
\text { Poole }\end{array}$ & $\begin{array}{l}\text { Centre for } \\
\text { Prevention of } \\
\text { Stroke and } \\
\text { Dementia, } \\
\text { University of } \\
\text { Oxford, UK }\end{array}$ & $\begin{array}{l}\text { Laboratory } \\
\text { technician }\end{array}$ & $\begin{array}{l}\text { Performed } \\
\text { laboratory } \\
\text { tests }\end{array}$ \\
\hline
\end{tabular}

Appendix 2 (continued)

\begin{tabular}{|c|c|c|c|}
\hline Name & Location & Role & Contribution \\
\hline $\begin{array}{l}\text { Julia } \\
\text { Duerden }\end{array}$ & $\begin{array}{l}\text { Centre for } \\
\text { Prevention of } \\
\text { Stroke and } \\
\text { Dementia, } \\
\text { University of } \\
\text { Oxford, UK }\end{array}$ & $\begin{array}{l}\text { Laboratory } \\
\text { technician }\end{array}$ & $\begin{array}{l}\text { Performed } \\
\text { laboratory } \\
\text { tests }\end{array}$ \\
\hline $\begin{array}{l}\text { Debbie } \\
\text { Green }\end{array}$ & $\begin{array}{l}\text { Centre for } \\
\text { Prevention of } \\
\text { Stroke and } \\
\text { Dementia, } \\
\text { University of } \\
\text { Oxford, UK }\end{array}$ & $\begin{array}{l}\text { Administrative } \\
\text { assistant }\end{array}$ & $\begin{array}{l}\text { Involved in } \\
\text { the follow-up } \\
\text { of patients }\end{array}$ \\
\hline $\begin{array}{l}\text { Maria } \\
\text { Drummond }\end{array}$ & $\begin{array}{l}\text { Centre for } \\
\text { Prevention of } \\
\text { Stroke and } \\
\text { Dementia, } \\
\text { University of } \\
\text { Oxford, UK }\end{array}$ & $\begin{array}{l}\text { Administrative } \\
\text { assistant }\end{array}$ & $\begin{array}{l}\text { Involved in } \\
\text { the follow-up } \\
\text { of patients }\end{array}$ \\
\hline
\end{tabular}

\section{References}

1. Amarenco P, Lavallee PC, Monteiro Tavares L, et al. Five-year risk of stroke after TIA or minor ischemic stroke. N Engl J Med 2018;378:2182-2190.

2. Boulanger M, Béjot $\mathrm{Y}$, Rothwell PM, Touzé E. Long-term risk of myocardial infarction compared to recurrent stroke after transient ischemic attack and ischemic stroke: systematic review and meta-analysis. J Am Heart Assoc 2018;7: e007267.

3. Hart RG, Sharma M, Mundl H, et al. Rivaroxaban for stroke prevention after embolic stroke of undetermined source. N Engl J Med 2018;378:2191-2201.

4. The Stroke Prevention in Reversible Ischemia Trial (SPIRIT) Study Group. A randomized trial of anticoagulants versus aspirin after cerebral ischemia of presumed arterial origin.Ann Neurol 1997;42:857-865.

5. Bhatt DL, Eagle KA, Ohman EM, et al. Comparative determinants of 4-year cardiovascular event rates in stable outpatients at risk of or with atherothrombosis. JAMA 2010;304:1350-1357.

6. Steg PG, Bhatt DL, Wilson PW, et al. One-year cardiovascular event rates in outpatients with atherothrombosis. JAMA 2007;297:1197-1206.

7. van den Berg MJ, Bhatt DL, Kappelle LJ, et al. Identification of vascular patients at very high risk for recurrent cardiovascular events: validation of the current ACC/AHA very high risk criteria. Eur Heart J 2017;38:3211-3218.

8. Eikelboom JW, Connolly SJ, Bosch J, et al. Rivaroxaban with or without aspirin in stable cardiovascular disease. N Engl J Med 2017;377:1319-1330.

9. Anand SS, Bosch J, Eikelboom JW, et al. Rivaroxaban with or without aspirin in patients with stable peripheral or carotid artery disease: an international, randomised, double-blind, placebo-controlled trial. Lancet 2018;391:219-229.

10. Hankey GJ. Secondary stroke prevention. Lancet Neurol 2014;13:178-194.

11. Li L, Yiin GS, Geraghty OC, et al. Incidence, outcome, risk factors, and long-term prognosis of cryptogenic transient ischaemic attack and ischaemic stroke: a population-based study. Lancet Neurol 2015;14:903-913.

12. Martinez-Majander N, Aarnio K, Pirinen J, et al. Embolic strokes of undetermined source in young adults: baseline characteristics and long-term outcome. Eur J Neurol 2018;25:535-541.

13. Mas JL, Derumeaux G, Guillon B, et al. Patent foramen ovale closure or anticoagulation vs. antiplatelets after stroke. N Engl J Med 2017;377:1011-1021.

14. Carroll JD, Saver JL, Thaler DE, et al. Closure of patent foramen ovale versus medical therapy after cryptogenic stroke. N Engl J Med 2013;368:1092-1100.

15. Amarenco P, Bogousslavsky J, Callahan A III, et al. High-dose atorvastatin after stroke or transient ischemic attack. N Engl J Med 2006;355:549-559.

16. Boekholdt SM, Hovingh GK, Mora S, et al. Very low levels of atherogenic lipoproteins and the risk for cardiovascular events: a meta-analysis of statin trials. J Am Coll Cardiol 2014;64:485-494.

17. Treat Stroke to Target. Available at: clinicaltrials.gov/ct2/show/NCT01252875? term=NCT01252875\&rank=1. Accessed October 27, 2018.

18. Ridker PM, Revkin J, Amarenco P, et al. Cardiovascular efficacy and safety of bococizumab in high-risk patients. N Engl J Med 2017;376:1527-1539.

19. Sabatine MS, Giugliano RP, Keech AC, et al. Evolocumab and clinical outcomes in patients with cardiovascular disease. N Engl J Med 2017;376:1713-1722.

20. HPS TIMI REVEAL Collaborative Group, Bowman L, Hopewell JC, et al. Effects of anacetrapib in patients with atherosclerotic vascular disease. N Engl J Med 2017;377: $1217-1227$.

21. O'Donnell MJ, Denis X, Liu L, et al. Risk factors for ischaemic and intracerebral haemorrhagic stroke in 22 countries (the Interstroke study): a case-control study. Lancet 2010;376:112-123. 
22. Yusuf S, Hawken S, Ounpuu S, et al. Effect of potentially modifiable risk factors associated with myocardial infarction in 52 countries (the Interheart study): casecontrol study. Lancet 2004;364:937-952.

23. Kazi DS, Moran AE, Coxson PG, et al. Cost-effectiveness of pcsk9 inhibitor therapy in patients with heterozygous familial hypercholesterolemia or atherosclerotic cardiovascular disease. JAMA 2016;316:743-753.

24. Rothwell PM, Coull AJ, Giles MF, et al. Change in stroke incidence, mortality, casefatality, severity, and risk factors in Oxfordshire, UK from 1981 to 2004 (Oxford Vascular Study). Lancet 2004;363:1925-1933.

25. Coull AJ, Silver LE, Bull LM, Giles MF, Rothwell PM, Oxford Vascular Study. Direct assessment of completeness of ascertainment in a stroke incidence study. Stroke 2004; 35:2041-2045.

26. Feigin V, Hoorn SV. How to study stroke incidence. Lancet 2004;363:1920.

27. Adams HP Jr, Bendixen BH, Kappelle LJ, et al. Classification of subtype of acute ischemic stroke: definitions for use in a multicenter clinical trial: TOAST: Trial of Org 10172 in Acute Stroke Treatment. Stroke 1993;24:35-41.

28. Levey AS, Coresh J, Greene T, et al. Expressing the modification of diet in renal disease study equation for estimating glomerular filtration rate with standardized serum creatinine values. Clin Chem 2007;53:766-772.

29. Myocardial infarction redefined: a consensus document of the Joint European Society of Cardiology/American College of Cardiology Committee for the redefinition of myocardial infarction. Eur Heart J 2000;21:1502-1513.

30. Luepker RV, Apple FS, Christenson RH, et al. Case definitions for acute coronary heart disease in epidemiology and clinical research studies: a statement from the AHA Council on Epidemiology and Prevention; AHA Statistics Committee; World Heart Federation Council on Epidemiology and Prevention; the European Society of Cardiology Working Group on Epidemiology and Prevention; Centers for Disease Control and Prevention; and the National Heart, Lung, and Blood Institute. Circulation 2003;108:2543-2549.

31. Yusuf S, Zhao F, Mehta SR, Chrolavicius S, Tognoni G, Fox KK. Effects of clopidogrel in addition to aspirin in patients with acute coronary syndromes without ST-segment elevation. N Engl J Med 2001;345:494-502.

32. Fonarow GC, Keech AC, Pedersen TR, et al. Cost-effectiveness of evolocumab therapy for reducing cardiovascular events in patients with atherosclerotic cardiovascular disease. JAMA Cardiol 2017;2:1069-1078.

33. Li L, Geraghty OC, Mehta Z, Rothwell PM. Age-specific risks, severity, time course, and outcome of bleeding on long-term antiplatelet treatment after vascular events: a population-based cohort study. Lancet 2017;390:490-499.

34. Barnett HJ, Taylor DW, Eliasziw M, et al. Benefit of carotid endarterectomy in patients with symptomatic moderate or severe stenosis: North American
Symptomatic Carotid Endarterectomy Trial Collaborators. N Engl J Med 1998; 339:1415-1425.

35. Randomised trial of endarterectomy for recently symptomatic carotid stenosis: final results of the MRC European Carotid Surgery Trial (ECST). Lancet 1998;351: 1379-1387.

36. Third report of the National Cholesterol Education Program (NCEP) expert panel on detection, evaluation, and treatment of high blood cholesterol in adults (Adult Treatment Panel III) final report. Circulation 2002;106:3143-3421.

37. Lackland DT, Elkind MS, D'Agostino R Sr, et al. Inclusion of stroke in cardiovascular risk prediction instruments: a statement for healthcare professionals from the American Heart Association/American Stroke Association. Stroke 2012;43:1998-2027.

38. Wijnhoud AD, Koudstaal PJ, Dippel DW. The prognostic value of pulsatility index, flow velocity, and their ratio, measured with TCD ultrasound, in patients with a recent TIA or ischemic stroke. Acta Neurol Scand 2011;124:238-244.

39. Boulanger M, Poon MT, Wild SH, Al-Shahi Salman R. Association between diabetes mellitus and the occurrence and outcome of intracerebral hemorrhage. Neurology 2016;87:870-878.

40. Diener HC, Easton JD, Granger CB, et al. Design of randomized, double-blind, evaluation in secondary stroke prevention comparing the efficacy and safety of the oral thrombin inhibitor dabigatran etexilate vs. acetylsalicylic acid in patients with embolic stroke of undetermined source (RE-SPECT ESUS). Int J Stroke 2015;10:1309-1312.

41. PROGRESS Collaborative Group. Randomised trial of a perindopril-based bloodpressure-lowering regimen among 6,105 individuals with previous stroke or transient ischaemic attack. Lancet 2001;358:1033-1041.

42. Hamann GF, Weimar C, Glahn J, Busse O, Diener H-C. Adherence to secondary stroke prevention strategies: results from the German Stroke Data Bank. Cerebrovasc Dis $2003 ; 15: 282-288$.

43. Hillen T, Dundas R, Lawrence E, Stewart JA, Rudd AG, Wolfe CD. Antithrombotic and antihypertensive management 3 months after ischemic stroke: a prospective study in an inner city population. Stroke 2000;31:469-475.

44. Mouradian MS, Majumdar SR, Senthilselvan A, Khan K, Shuaib A. How well are hypertension, hyperlipidemia, diabetes, and smoking managed after a stroke or transient ischemic attack? Stroke 2002;33:1656-1659.

45. Ovbiagele B, Saver JL, Fredieu A, et al. In-hospital initiation of secondary stroke prevention therapies yields high rates of adherence at follow-up. Stroke 2004;35:2879-2883.

46. Hemingway H, Shipley M, Britton A, Page M, Macfarlane P, Marmot M. Prognosis of angina with and without a diagnosis: 11 year follow up in the Whitehall II prospective cohort study. BMJ 2003;327:895.

47. Thygesen K, Alpert JS, Jaffe AS, et al. Fourth universal definition of myocardial infarction (2018). Eur Heart J 2019;40:237-269. 


\section{Neurology}

\section{Effect of coexisting vascular disease on long-term risk of recurrent events after TIA or stroke}

Marion Boulanger, Linxin Li, Shane Lyons, et al. Neurology 2019;93;e695-e707 Published Online before print July 23, 2019

DOI 10.1212/WNL.0000000000007935

\section{This information is current as of July 23, 2019}

\section{Updated Information \&} Services

\section{References}

Subspecialty Collections

Permissions \& Licensing

Reprints including high resolution figures, can be found at: http://n.neurology.org/content/93/7/e695.full

This article cites 46 articles, 13 of which you can access for free at: http://n.neurology.org/content/93/7/e695.full\#ref-list-1

This article, along with others on similar topics, appears in the following collection(s):

\section{All Cerebrovascular disease/Stroke}

http://n.neurology.org/cgi/collection/all_cerebrovascular_disease_strok e

Cardiac

http://n.neurology.org/cgi/collection/cardiac

Cohort studies

http://n.neurology.org/cgi/collection/cohort_studies

Prognosis

http://n.neurology.org/cgi/collection/prognosis

Stroke prevention

http://n.neurology.org/cgi/collection/stroke_prevention

Information about reproducing this article in parts (figures,tables) or in its entirety can be found online at:

http://www.neurology.org/about/about_the_journal\#permissions

Information about ordering reprints can be found online:

http://n.neurology.org/subscribers/advertise

Neurology ${ }^{\circledR}$ is the official journal of the American Academy of Neurology. Published continuously since 1951, it is now a weekly with 48 issues per year. Copyright Copyright (C) 2019 The Author(s). Published by Wolters Kluwer Health, Inc. on behalf of the American Academy of Neurology.. All rights reserved. Print ISSN: 0028-3878. Online ISSN: 1526-632X.

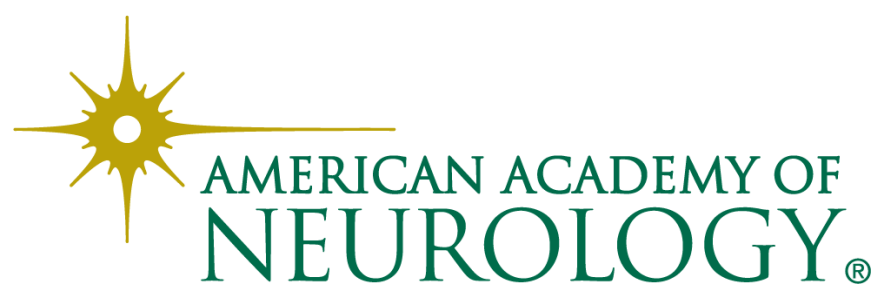

